\title{
Rasch Analysis in Understanding Mathematics Anxiety
}

\author{
Jared Jorim O. Mendoza*, James Roldan S. Reyes and Joyce D. L. Grajo \\ University of the Philippines Los Banos, Institute of Statistics; jomendoza1@up.edu.ph, jsreyes7@up.edu.ph, \\ jdgrajo@up.edu.ph
}

\begin{abstract}
This paper aims to understand students' mathematics anxiety through determining specific areas that significantly influence this sort of feeling towards mathematics subject. Setting the application of Rasch techniques, a survey data from a 32-item questionnaire, all in Likert-scale type, was used. The questionnaire is divided into four specific areas namely peer, test/assessment, teacher, and computational/numerical factors. Participants of the survey consist of a random sample of high school students who were equally allocated across grade level. A dichotomous Rasch model was fitted and the differential item functioning was then applied. Research findings revealed that student's mathematics anxiety could be lessened or if possible could be avoided by: (1) Encouraging a class success rather than an individual success within and outside classroom, (2) Guiding student learning under the teacher's availability and approachability; (3) Considering student's preparedness prior the conduct of any learning assessment, and (4) Designing assessment tools that are both accurate and appropriate. Moreover, this paper has identified the different roots of students' mathematics anxiety which include sex, study time in mathematics, whether or not mathematics is the favorite subject, and the type of learning by the students. The results of this paper can further be used by mathematics educators to gain insights about these causes of mathematics anxiety making classroom instruction effective as well as the learning of the subject both meaningful and fun to the students. In general, the techniques applied in this paper could be adopted in other discipline to understand more the student's behavior towards a particular subject.
\end{abstract}

Keywords: Item Response Model, Likert-Scale Items, Mathematics Anxiety, Rasch Scores, Rasch Techniques

\section{Introduction}

Nobody can refute the significance of mathematics to people's everyday life. From financial transactions to other areas of profession, whether simple or complex, the concept of mathematics cannot be neglected. A lot of scientific theories and laws are derived from mathematical formulas, bringing mathematics as the language of science. However, despite the application of mathematics in various fields, people usually find the discipline as a dreary course. Most of them feel a negative reaction, a sense of tension, and a mood of anxiousness that impede them in solving numerical problems, in both classroom and realworld settings. This state is known as mathematics anxiety. Tobias and Weissbrod" define mathematics anxiety as "the panic, helplessness, paralysis, and mental disorganization that arises among some people when they are required to solve a mathematical problem". Moreover, in ${ }^{2}$, it was found that having mathematics anxiety is a factor that affects student achievement. In fact, differences could be observed in student achievement based on the level of mathematics anxiety. Reali et al. examined the link of mathematics anxiety and mathematics performance among students in Colombia ${ }^{3}$. Their paper found that an inverse relationship exists between performance in mathematics and mathematics anxiety. The results of their paper also indicate that this negative relationship is found to be stronger in female students than their male contemporaries.

Commonly, if Filipino students are asked about his least favorite subject, it is more likely to get mathematics as a response. In 2011, the Philippine education ranked

${ }^{*}$ Author for correspondence 
poor based on 2010-2011 Global Competitiveness Report of the World Economic Forum ${ }^{4}$. In primary education, the Philippines took the 99th spot out of 138 countries and ranked 112th in science and mathematics. Furthermore, based on the Philippines' last participation in the Trends in International Mathematics and Science (TIMMS) in 2003, the country placed in the bottom four among 38 participating countries in Grade 8 mathematics and placed second to the last among 25 participating countries in Grade 4 mathematics ${ }^{5}$. This low performance in mathematics among Filipino students could be accounted by their level of mathematics anxiety.

Measuring attitudes and behavior, like mathematics anxiety, through a survey often requires a series of questions to evaluate them effectively. Usually, these series of questions, commonly in Likert-scale or in dichotomous type, are summed up to generate a single numerical index that could capture an overall measure of an individual's attitude or behavior for a certain investigation. Multivariate analyses, like factor analysis (FA), are commonly applied statistical techniques in analyzing this type of data. Although FA evaluates the dimensionality of a data set, it still ignores the fact that the series of questions is intended to measure a single construct. FA is more commonly used to identify multiple sources of variance from a single latent trait data set. This study makes use of a 32-item questionnaire to study dimensionality of mathematics anxiety along with evidence of local dependence, interactions of questionnaire items with sample characteristics, and item fit. This cannot be done by FA alone; hence, the application of Rasch analysis is explored.

Rasch Analysis is an item response theory (IRT) model in measuring quantitative latent variable, the overall anxiety score, in particular, on a metric level. It has the properties of separability, sufficiency, specific objectivity, latent additivity, and invariant property of estimates ${ }^{6}$. If the invariance property is not met, Rasch analysis is useful to evaluate differences among students belonging to a certain profile. This can be done using the differential functioning item (DIF). Several articles compared Rasch analysis with the use of FA. According to the research in ${ }^{7}$, FA is based on the correlation of an item with other items to load that item for a factor, which is in contrast with Rasch. In Rasch analysis, the items construct hierarchical structure where positive responses to difficult items imply positive responses to easier items, but not vice versa. Sick also mentioned the difficulty of FA in analyzing Likert data. "When an item is difficult to endorse, it may not correlate strongly with items that are easy to endorse, even if these items are indicative of the same trait. In some instances, easy items and difficult items may not load together, forming difficulty factors, a misleading result

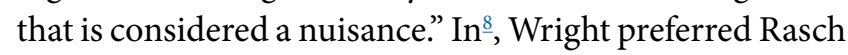
measurement over FA for reducing complex data matrices to unidimensional variables since FA can mistakenly construct linear measurement for ordinal observations especially in likert type. He further developed procedures on how to apply Rasch measurement as replacement in FA.

The idea in ${ }^{9}$ applied Rasch analysis in measuring financial literacy among students. This is the first attempt to apply Rasch model in the domain of financial literacy testing. Results of their study identified areas and groups which showed largest knowledge deficiency in finance. Another study used Rasch analysis in measuring service quality and customer satisfaction ${ }^{10}$. They concluded that Rasch model is a good method if it is aimed to obtain the probabilistic individual satisfaction measure per customer and a specific quality measure for each item.

With the use of Raschanalysis, this paperidentifiesareas that cause students to have mathematics anxiety. Further, the paper identifies which group of students demonstrates highest mathematics anxiety. Understanding these roots of mathematics anxiety is beneficial especially for the Philippine educational system since this will lead for the creation of methods and solutions that could enhance the country's mathematics education and at the same time, could increase the interest of Filipino students in the area of mathematics as their discipline and profession.

\section{Methodology}

The questionnaire comprised of 32 questions, divided into four specific areas: (1) Peer factor, (2) Test/assessment factor, (3) Teacher factor, and (4) Computational/ numerical factor. Each area has eight set of questions answered in a 5-point scale, with 1 being the lowest and 5 being the highest. Also, included in the questionnaire are variables for student profiling in which frequency analysis is done. The survey was conducted on a stratified random sample of 108 UP Rural High School students registered in the academic year 2016-2017. Samples are equally allocated among Grade 7, Grade 8, Grade 9, and 
Grade 10 students. The data was obtained through a self-administered survey.

Responses to each item were recoded to low (levels 1-3) and high (levels 4-5) anxiety to fit the dichotomous Rasch model

$$
B_{n}-D_{i}=1 \mathrm{n}\left(\frac{P_{n i}}{1-P_{n i}}\right)
$$

where $B_{n}$ is the measure of mathematics anxiety of the student across all items in the questionnaire; $D_{i}$ is the measure of how much the item in the questionnaire triggers mathematics anxiety, $P_{n i}$ is the probability of the student to have high mathematics anxiety in an item in the questionnaire, $1-P_{n i}$ is the probability of the student to have low mathematics anxiety in an item in the questionnaire.

Rasch analysis is employed to each of the four dimensions of mathematics anxiety on the assumption that the dimensions contribute differently to the overall anxiety score. Differential item functioning is then used to determine whether certain subgroups of students have higher anxiety over the other. Finally, raw scores and scores from the Rasch models are compared in each dimension.

\section{Results and Discussion}

Table 1 reports the summary test-of-fit statistics for the Rasch model in the four dimensions of mathematics anxiety. The power of test-of-fit of the models are average to good with separation indices ranging from 0.60 to 0.79. Similar to reliability indices such as Cronbach's $\alpha$, separation indices of this value mean that the proportion of person variance that is not due to error is high and thus, the models are fairly reliable.

As responses are coded 0 for low anxiety and 1 for high anxiety, the item difficulty parameter in this study measures the gravity of a student's mathematics anxiety. Figure 2 shows

Table 1. Summary of the test of fit statistics for Rasch model in each dimension

\begin{tabular}{lccc}
\hline \multicolumn{1}{c}{ Dimension } & $X^{2}$ & p-value & $\begin{array}{c}\text { Separation } \\
\text { Index }\end{array}$ \\
\hline Peer & 10.66 & 0.154 & 0.67 \\
Test/Assessment & 8.28 & 0.218 & 0.79 \\
Teacher & 7.08 & 0.314 & 0.60 \\
Computational/ & 6.85 & 0.335 & 0.73 \\
Numerical & & &
\end{tabular}
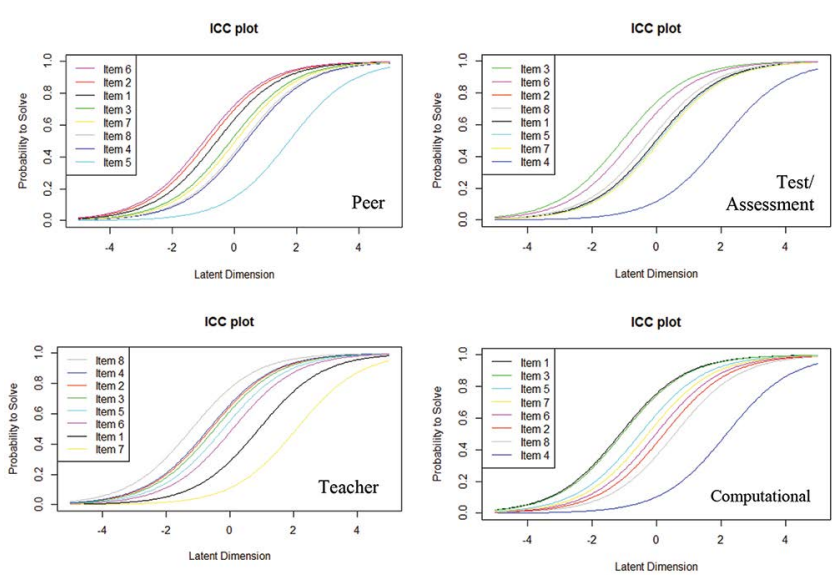

Figure 1. Item characteristic curve for the questionnaire items in each dimension.

the item characteristic curve (ICC) for the questionnaire items in the four dimensions. Comparing the ICCs, it can be observed in peer dimension that hearing classmates talk about their answers in a quiz/exam (item 6) and classmates having high scores in a quiz/exam (item 2) cause high anxiety while having a group review with friends causes minimal anxiety (item 5). In teacher dimension, a math teacher speaking very fast triggers high anxiety among students (item 8 ) while hearing a math teacher being present at school (item 1) and seeing a math teacher outside school (item 7) contribute very little to their anxiety. Items 3 and 6 in assessment dimension: announcing a sudden surprise quiz and each question being full points or nothing are sources of high anxiety in students but announcing the coverage for an exam (item 4) is not. In computational/numerical dimension, solving a complicated problem (item 1) and having computed answer not among the choices in a quiz/exam (item 3 ) generate high anxiety whereas inputting values in a calculator (item 4 ) produces low mathematics anxiety in students.

The frequency analysis tells that most students are female (55\%); they choose mathematics as favorite subject (33.33\%); and they are visual learners (65\%). Also, it was found that $50 \%$ of the students spend studying mathematics above 2.5 hours per week. Using these students' profile, interestingly, the Rasch model was able to distinguish differences in anxiety levels between subgroups of students. Differential item tests among student subgroups are reported in Table 2. Comparisons between subgroups further reveal that visual learners get more anxious when there are several sub-items in quiz/ exam questions and when solving complicated math problems. Female students tend to have higher anxiety 
Table 2. Differential item test among student subgroups

\begin{tabular}{lcc}
\hline \multicolumn{1}{c}{ Item } & $Z$ & -value $^{*}$ \\
\hline SEX & & 0.091 \\
Trying to ask a question to the math teacher (T) & -1.690 & 0.065 \\
Studying for a quiz/exam (A) & 1.846 & \\
MATH AS FAVORITE SUBJECT & & 0.049 \\
Friends and classmates being good at math (P) & -1.973 & 0.016 \\
Classmates having high scores in quiz/exam (P) & -2.399 & 0.030 \\
Announcing a sudden surprise quiz (A) & 2.174 & 0.072 \\
Memorizing formulas (C) & -1.799 & \\
TIME ALLOTED FOR MATH REVIEW & & 0.073 \\
Each question being all or nothing (A) & 1.796 & 0.010 \\
Solving a complicated problem (C) & -2.581 & 0.060 \\
IF VISUAL LEARNER & & 0.047 \\
Math teacher being very serious (T) & 1.884 & 0.005 \\
Many sub-items being asked (A) & -1.985 & 0.032 \\
Memorizing formulas (C) & 2.839 & \\
Solving a complicated problem (C) & -2.143 & 0.086 \\
IF HANDS-ON LEARNER & & 0.095 \\
Classmates having high scores in a quiz/exam (P) & & 0.039 \\
When hearing classmates move on to the next page of exam (P) & -1.718 & 0.093 \\
Math teacher being very serious (T) & -1.672 & 0.006 \\
Consulting with a math teacher (T) & -2.069 & 0.045 \\
Memorizing Formulae (C) & 1.681 & 0.040 \\
Solving a complicated problem (C) & -2.744 & 2.008 \\
Solving a problem in the board (C) & 2.055 & \\
\hline P & & \\
\hline
\end{tabular}

$\mathrm{P}$ - peer factor, $\mathrm{A}$ - test/assessment factor, $\mathrm{T}$ - teacher factor, $\mathrm{C}$ - computational/numerical factor

${ }^{\star}$ Using $10 \%$ level of significance

when trying to ask a question to their math teacher while male students tend to have higher anxiety when studying for a quiz/exam. Higher anxiety is observed in students whose favorite subject is math when their friends and classmates are good at the subject having high scores in quizzes/exams. They also feel more anxious when memorizing formulas. Announcement of a sudden surprise quiz, on the other hand, brings higher anxiety to students whose favorite subject is not math. Students who spend more than average time reviewing for math are less anxious even when questions in the exam are worth full points or nothing. Intriguingly, this group of students gets more anxious when faced with complicated math problems. On the other hand, students who are hands-on learners face higher anxiety when their classmates have high scores in a quiz/exam and when they hear their classmates move on to the next page during exam. They also tend to be more anxious when memorizing formulae and when their teacher is being too serious. For those students who are not hands-on learners, a higher anxiety happens when they consult with a math teacher, when they solve a complicated problem, and when they solve the problem in board. However, it should be noted that ineffective and inefficient studying styles were not accounted in this study, variables that may affect the outcome of this particular differential analysis.

Frequency distributions of the raw scores and the scores generated using Rasch measure were constructed for every dimension. As seen in Figure 2, the distribution of raw scores for all dimensions is skewed towards the lower end, implying that most students have low mathematics anxiety. This is also exhibited in the 


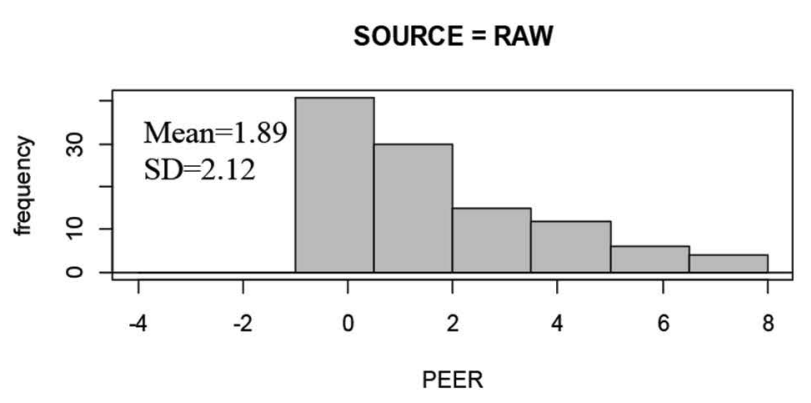

SOURCE $=$ RAW

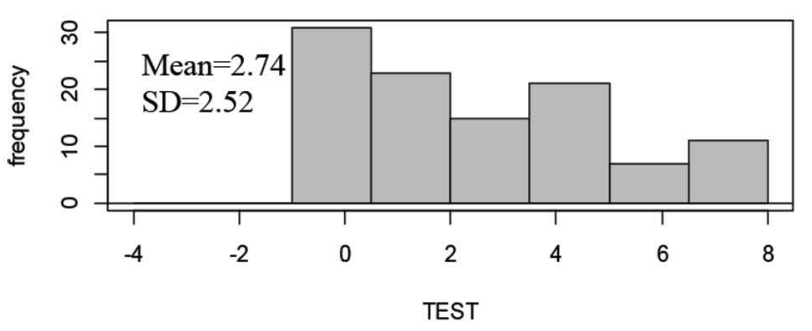

SOURCE $=$ RAW
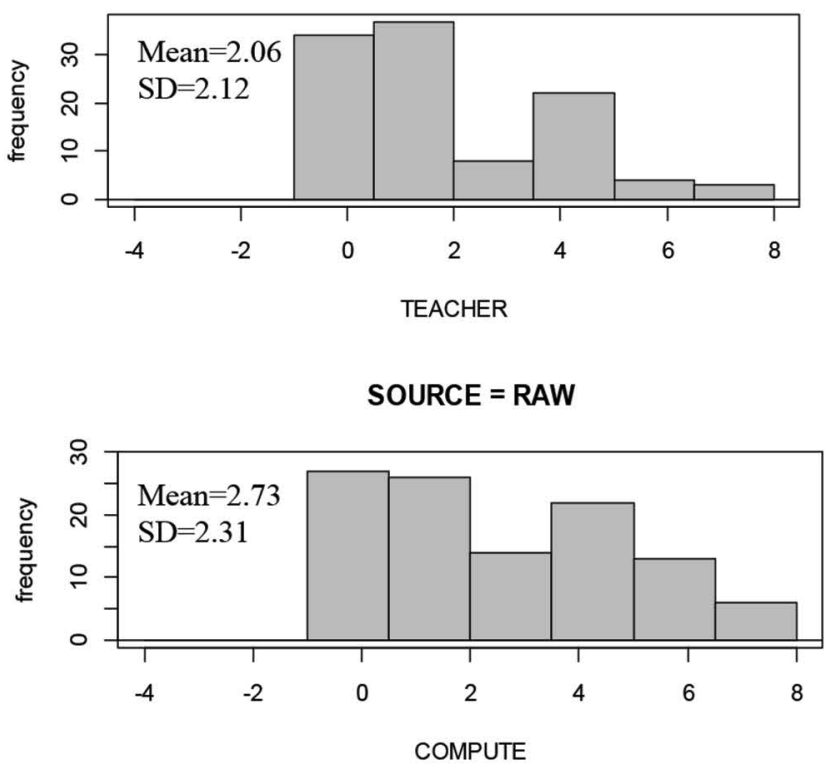

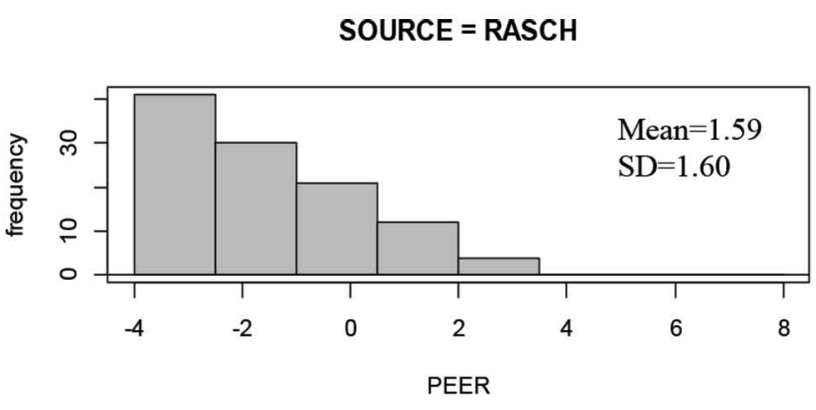

SOURCE $=$ RASCH

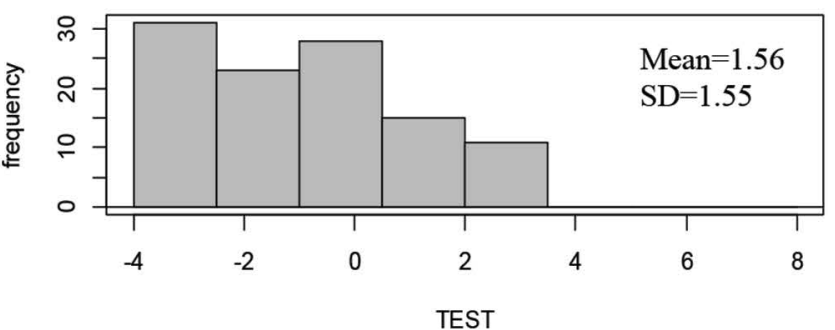

SOURCE $=$ RASCH

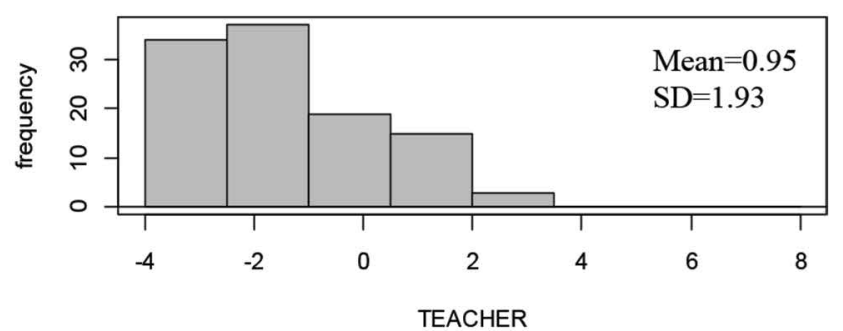

SOURCE $=$ RASCH

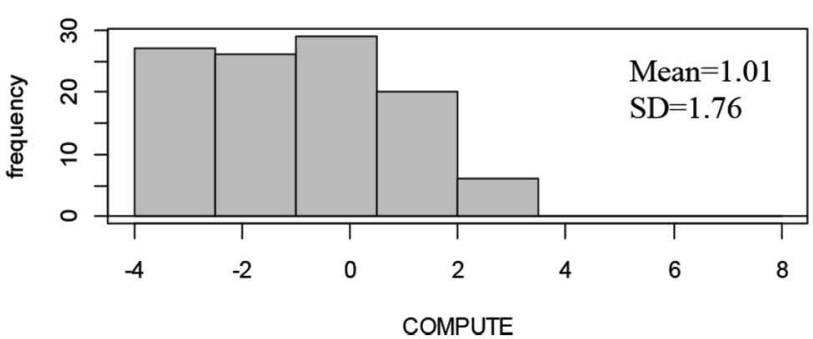

Figure 2. Frequency distribution of raw (left) and Rasch model (right) scores in each dimension.

distribution of scores using Rasch measure, although the mean shifts from positive to negative and the variability of scores decreases for all dimensions. Moreover, the correlation between raw score and score using Rasch measure has a value above 0.99 in all dimensions (Peer $=0.9956$, Test $/$ Assessment $=0.9964$, Teacher $=0.9957$, and Computational/Numerical $=0.9963$ ). This result is expected due to the fact that raw scores are the sufficient statistics for the Rasch measure. In ${ }^{11}$, Wright and Linacre mentioned that when the correlations are above 0.99 , the raw scores approximate linearity. This is usually achieved for data set with no missing data and no omitted cases, similar to the data set used in this paper. 


\section{Conclusion and Recommendations}

Though summing item responses can generate an overall score, this method does not take into account the effect of student's characteristics and the unequal contribution of each item with the overall measure. This leads the authors to explore the application of Rasch analysis in coming up an overall measure of mathematics anxiety score of the students.

With the identified key contributor of high mathematics anxiety for each dimension namely peer, test/assessment, teacher, and computational/ numerical anxiety, the pedagogy in mathematics education of the country can be now revisited and be further improved. Based on the results, student's mathematics anxiety could be avoided, if not possible, be decreased by doing the following: (1) Encouraging a class success rather than an individual success within and outside classroom; (2) Guiding student learning under the teacher's availability and approachability; (3) Considering student's preparedness prior the conduct of any learning assessment, and (4) Designing assessment tools that are both accurate and appropriate. This paper also found that certain subgroups of students are more anxious compared to the other. In this regard, educators of mathematics should completely understand and consider these students' differences to make learning of mathematics fun for the students and at the same time, encourage their active learning in the subject.

Moreover, the techniques applied in this study could be adopted in other discipline to understand more the student's behavior towards that subject. Also, exploring linkage of anxiety scores using Rasch measure with the mathematics grade of the students must be done. The authors recommend for a construction of an overall individual mathematics anxiety measure by obtaining the linear combinations of the overall score for each of the four dimensions.

\section{References}

1. Tobias S, Weissbrod C. Anxiety and mathematics: an update. Harvard Educational Review. 1980; 50(1):63-70. https://doi.org/10.17763/haer.50.1.xw483257j6035084

2. Effandi Z, Zain NM, Amalina N. Mathematics Anxiety and Achievement among Secondary School Students. American Journal of Applied Sciences. 2012; 9(11):1828-32. https://doi.org/10.3844/ajassp.2012.1828.1832

3. Reali F, Jimenez-Leal W, Maldonado-Carre-O, Devine A, Szcus D. Examining the Link between Math Anxiety and Math Performance in Colombian Students. Revista Colombiana de Psicologia. 2016; 25(2):369-79. https://doi.org/10.15446/rcp.v25n2.54532

4. De Leon MV. Philippine Education Ranked 'Poor'. Business Mirror. 2011. Available from: http://news. abs-cbn.com/business/06/14/11/philippine-educationranked-poor.

5. Capati RNA, Lapinid MRC. Assessing the Mathematics Performance of Grade 8 Students as Basis for Enhancing Instruction and Aligning with $\mathrm{K}$ to 12 Curriculum. Proceedings DLSU Research Congress. 2015; 3:1-7.

6. Rost J. The Growing Family of Rasch Models. Chapter 2. Essays on Item Response Theory. 2000; p. 25-42.

7. Sick J. Rasch Measurement and factor Analysis. SHIKEN: JALT Testing and Evaluation SIG Newsletter. 2011; 15(1):15-7.

8. Wright BD. Comparing Rasch Measurement and Factor Analysis. Structural Equation Modeling: A Multidisciplinary Journal. 2009; 1(3):3-24.

9. Bongini P, Trivellato P, Zenga M. Measuring Financial Literacy Among Students: An Application of Rasch Analysis. Electronic Journal of Applied Statistical Analysis. 2012; 5(3):425-30.

10. De Battisti F, Nicolini G, Salini S. The Rasch Model in Customer Satisfaction Survey Data. Quality Technology and Quantitative Management. 2010; 7(1):15-34. https://doi.org/10.1080/16843703.2010.11673216

11. Wright BD, Linacre JM. Differences between scores and measures. Rasch Measurement Transactions. 1989; $3(3): 63$. 